\title{
Effect of Increased Soil Fertility on the Yield and Energy Value of Short-Rotation Woody Crops
}

\author{
Mariusz J. Stolarski • Michal Krzyżaniak • Stefan Szczukowski • \\ Józef Tworkowski • Dariusz Załuski • Arkadiusz Bieniek • \\ Janusz Golaszewski
}

Published online: 28 December 2014

(C) The Author(s) 2014. This article is published with open access at Springerlink.com

\begin{abstract}
Biomass is produced as a feedstock for energy generation and industrial processes from short-rotation woody crop plantations in Europe, the USA, and Canada. This study determined the impact of soil enrichment on the survival rate, productivity, energy value, and yield of three species of crops grown on poor soil in a 4-year harvest rotation based on two factors: species (willow, poplar, and black locust) and fertilization (lignin, mineral fertilization, mycorrhiza inoculation, and their combination). The highest average yield was obtained from willow, followed by poplar and black locust. The highest yield in the entire experiment was for poplar with lignin combined with mineral fertilization (10.5 odt $\mathrm{ha}^{-1}$ year $^{-1}$ ). Using lignin combined with mineral fertilizers increased the yield by $8-14 \%$ compared to mineral fertilizers alone for willow and poplar and nearly doubled the black locust yield. The energy value of the yield ranged from 28.6 to $176.7 \mathrm{GJ} \mathrm{ha}^{-1}$ year $^{-1}$, respectively, for black locust grown on the control plot and for poplar grown with mineral fertilization combined with lignin.
\end{abstract}

Keywords Willow · Poplar · Black locust · Fertilization · Yield · Yield energy value

\footnotetext{
M. J. Stolarski $(\bowtie) \cdot M$. Krzyżaniak • S. Szczukowski •

J. Tworkowski $\cdot$ D. Załuski $\cdot$ J. Gołaszewski

Department of Plant Breeding and Seed Production, Faculty of Environmental Management and Agriculture, University of Warmia and Mazury in Olsztyn, Plac Łódzki 3/420, 10-724 Olsztyn, Poland e-mail: mariusz.stolarski@uwm.edu.pl

A. Bieniek

Department of Soil Science and Soil Protection, Faculty of Environmental Management and Agriculture, University of Warmia and Mazury in Olsztyn, Olsztyn, Poland
}

\section{Introduction}

Biomass as feedstock for energy generation and industrial processes on short-rotation woody crop (SRWC) plantations is being produced in many countries of Europe [1-5], the USA, and Canada [6-8]. Crops grown in the SRWC system include poplar, willow, and black locust. Poplar is grown mainly in the southern regions of Europe [9-11] and willow in the northern regions. Willow is grown on the largest area in Sweden-about 12,000 ha [1]. This species is also grown in areas exceeding 5,000 ha in Poland and Denmark and between 2,000-4,000 ha in Germany and the UK [12]. However, Hungary has the largest area of black locust plantations, and attempts have been made to grow it as a short-rotation crop for biomass production $[13,14]$.

SRWC plantations are often established on poor marginal soils (dry, damp, or with poor location) or contaminated soils (unsuitable for the cultivation of edible crops or fodder). Crops used for energy generation or industry should not compete with food production. Nevertheless, plantation owners producing biomass for commercial purposes will seek the highest yield per unit area. Several studies have shown that the yield of SRWC biomass is affected by a number of interrelated factors, including species, cultivar, and cultivation clone $[2,7,15,16]$. Soil conditions are also a major factor $[2$, 17]. Other factors which have an impact on the yield of SRWC include planting density and harvest frequency [3, 18], climatic conditions, and agricultural procedures [19-21]. When SRWC are grown on soils of poor quality, the type and dose of fertilizers used are of great importance [1,22], although some studies have shown the impact of this factor on the yield to be limited [23-25]. In the majority of studies, the effect of mineral fertilization has been compared to fertilization with animal manure or sludge, although there is limited data regarding the effect of soil enrichment by mycorrhizal inoculation or lignin on the productivity of SRWC compared to 
mineral fertilization. Mycorrhiza may increase the biomass production of SRWC, improve tolerance of abiotic and biotic stress, and increase resistance against soil pathogens [26]. Large volumes of lignin are available in the market; in 2010, the production was approximately 50 million tonnes of extracted lignin, but only 1 million tonne was commercially used for low-value products, the rest was burnt as a low-value fuel [27]. Therefore, low-purity lignin may also find application in improving soil structure and increasing the content of organic carbon to enhance the plant development conditions and their yield. Further, this study sought to determine the effect of soil enrichment on the survival rate, morphological features, yield, and energy value of three species of plants grown on a poorsoil site with low suitability for edible crops.

\section{Materials and Methods}

\section{Soil Characteristics}

The experiment was located in northeastern Poland $\left(53^{\circ} 59^{\prime} \mathrm{N}\right.$, $21^{\circ} 04^{\prime}$ E) on an experimental field owned by the University of Warmia and Mazury in Olsztyn (UWM). The study area was situated in varied undulating terrain, although the area of the experimental field was relatively flat. The soil analysis showed that the experiment was set up in brunic arenosol (Dystric) soil, formed from sand (Table 1). The soil was periodically too dry, and the level of underground water was below $150 \mathrm{~cm}$. The mesopore content in its surface layers (which is indicative of the amount of water available to plants) in slightly loamy sand was low, and it was very low in the underlying sand. Macropores (19.3-30.4\%) dominated the entire soil profile.

\section{Setting Up and Conducting the Experiment}

Winter triticale $(\times$ Triticosecale Wittm. ex A. Camus) was grown in rotation as forecrop for SRWC. Roundup spray was applied at $5 \mathrm{dm}^{3} \mathrm{ha}^{-1}$ after the triticale was harvested. Subsequently, after about 3 weeks, discing of the soil was done, and deep ploughing at a depth of $30 \mathrm{~cm}$ was done in late autumn 2009. In the second week of April 2010, the field was disced and harrowed, places for planting were marked out, and cuttings of willow and poplar and seedlings of black locust were planted manually. The cuttings were $25 \mathrm{~cm}$ in length, and their diameter was $0.9-1.1 \mathrm{~cm}$, whereas rooted seedlings of black locust were about $30-35 \mathrm{~cm}$ in height.
Table 1 Soil texture and physico-chemical parameters of soil

\begin{tabular}{|c|c|c|c|c|}
\hline \multirow[t]{2}{*}{ Parameter } & \multirow[t]{2}{*}{ Unit } & \multicolumn{3}{|l|}{ Horizon $(\mathrm{cm})$} \\
\hline & & $\begin{array}{l}\text { A } \\
(0-21)\end{array}$ & $\begin{array}{l}\text { Bv } \\
(21-41)\end{array}$ & $\begin{array}{l}\mathrm{C} \\
(41-150)\end{array}$ \\
\hline Macropores & $\varnothing>30 \mu \mathrm{m}$ & 19.30 & 22.75 & 30.42 \\
\hline Mesopores & $\varnothing=30-0.2 \mu \mathrm{m}$ & 14.80 & 15.82 & 10.32 \\
\hline Micropores & $\varnothing<0.2 \mu \mathrm{m}$ & 1.82 & 3.42 & 1.97 \\
\hline $\mathrm{pH}(\mathrm{KCl})$ & - & 7.05 & 6.30 & 7.92 \\
\hline \multirow[t]{2}{*}{ Organic matter } & $\%$ & 2.89 & - & - \\
\hline & $\mathrm{t} \mathrm{ha}^{-1}$ & 114.1 & - & - \\
\hline $\mathrm{N}-$ mineral & $\mathrm{mg} \mathrm{kg}^{-1}$ & 3.90 & 1.10 & 0.51 \\
\hline $\mathrm{P}$ & $\mathrm{mg} \mathrm{kg}^{-1}$ & 112.2 & 67.3 & 22.0 \\
\hline K & $\mathrm{mg} \mathrm{kg}^{-1}$ & 106.2 & 79.7 & 21.6 \\
\hline $\mathrm{Mg}$ & $\mathrm{mg} \mathrm{kg}^{-1}$ & 66.0 & 76.0 & 23.0 \\
\hline B & $m g \mathrm{~kg}^{-1}$ & 6.3 & 3.3 & 2.8 \\
\hline $\mathrm{Cu}$ & $\mathrm{mg} \mathrm{kg}^{-1}$ & 2.1 & 1.9 & 1.2 \\
\hline $\mathrm{Zn}$ & $\mathrm{mg} \mathrm{kg}^{-1}$ & 21.5 & 7.2 & 4.9 \\
\hline $\mathrm{Fe}$ & $\mathrm{mg} \mathrm{kg}^{-1}$ & 1360.0 & 1380.0 & 470.0 \\
\hline Soil texture & & Slightly loamy sand & Slightly loamy sand & Loose sand \\
\hline Clay & $\%$ & 2 & 1 & 0 \\
\hline$<0.002 \mathrm{~mm}$ & & & & \\
\hline Silt & $\%$ & 8 & 9 & 4 \\
\hline $\begin{array}{l}0.002-0.05 \mathrm{~mm} \\
\text { Sand }\end{array}$ & $\%$ & 90 & 90 & 96 \\
\hline $0.05-2.0 \mathrm{~mm}$ & & & & \\
\hline
\end{tabular}


Subsequently, after the cuttings of willow and poplar were planted, a solution of soil herbicide Guardian Complete MIX $664 \mathrm{SE}$ with water in $3.5: 300 \mathrm{dm}^{3} \mathrm{ha}^{-1}$ ratio was applied. No herbicide spray was used on the black locust plots. Mechanical weeding was performed three times (last weeks of May, June, and July) during the 2010 growing season.

This study was based on a two-factorial experiment. Three plant species, willow, poplar, and black locust, were the first experiment factor. The willow Salix viminalis L., clone UWM 006, was acquired from the UWM. Poplar Populus nigra $\times$ Populus maximowiczii Henry cv. Max-5 was provided by a farm in the north of Austria. Black locust (Robinia pseudoacacia L.), a native species, was provided by a forest nursery in Poland.

The other experimental factor was the fertilization method. The following options were identified: lignin (L), mineral fertilization $(\mathrm{F})$, mycorrhiza inoculation $(\mathrm{M})$, lignin + mineral fertilization (LF), mycorrhiza + mineral fertilization (MF), lignin + mycorrhiza (LM), lignin + mycorrhiza + mineral fertilization (LMF), and a control plot with no soil enrichment (C). The experiment was set up in three replications using 18.0- $\mathrm{m}^{2}$ plots.

Willow cuttings were planted in the conventional twin-row design with a spacing of $0.75 \mathrm{~m}$ within twin rows with $1.5 \mathrm{~m}$ between pairs of rows. Cuttings were spaced $0.8 \mathrm{~m}$ apart within the rows, with 11,000 plants per hectare.

Lignin (a waste product in the process of paper production) was applied in spring (before the experiment) at $13.3 \mathrm{tha}^{-1}$. It was scattered on the soil surface with a rear-discharge manure spreader before the discing and harrowing, which effectively mixed it with soil. The lignin applied in the experiment contained $61.72 \%$ organic matter and had acidic $\mathrm{pH}(4.1$ in $\mathrm{KCl})$.

Live mycorrhizal mycelium was applied, separately for each species, in early September 2010, after the willows, poplars, and black locusts had formed sufficient root systems. Inoculation in the form of liquid suspension at $30-35 \mathrm{~cm}^{3}$ was applied under each plant with a manual applicator (a manual sprayer with a special nozzle for inoculation application into the soil at a depth of $20 \mathrm{~cm}$ ). In general, one soil injection was made next to each plant so that the inoculation was introduced as close to the root system as possible.

Live mycelium was obtained by reproducing fungi isolated from the roots of Salix caprea which grows in Poland, concluding that this helped the willow plants to survive in the harsh conditions of light sandy soil. Genus Salix (including species of Salix viminalis) is capable of entering into symbiosis with many fungal species, both ectomycorrhizal and endomycorrhizal. For this reason, isolates were used which were the only ones available on the market originating from deeply mycorrhized roots of $S$. caprea. Poplar trees were inoculated with mycelium isolated from the roots of poplar grown as feedstock for energy generation in Spain. Since the plantation was inoculated with an isolate of poplar which grows in Poland, this represented a re-inoculation. The inoculation for black locust was a mixture of mycorrhizal fungi used in forest nurseries. Black locust does not have any species-specific mycorrhiza, and its roots are associated with many fungus species.

No top dressing was applied in the first year of growth because of the slow growth of the plant root systems. However, phosphorus and potassium were spread by hand before the second growing season (2011). Phosphorus was applied at $13 \mathrm{~kg} \mathrm{ha}^{-1}$ as a triple superphosphate. Potassium was applied at $50 \mathrm{~kg} \mathrm{ha}^{-1}$ as potassium salt. Nitrogen was applied in two doses. The first dose was applied as ammonium nitrate at $50 \mathrm{~kg} \mathrm{ha}^{-1}$ immediately before the start of the 2011 growing season. The second nitrogen dose in the same form was applied in mid-June $2011\left(40 \mathrm{~kg} \mathrm{ha}^{-1}\right)$.

Biometric Measurements, Determination of Biomass Yield, and Its Energy Value

After the fourth year of growth (2013), the plant density in each plot (per $1 \mathrm{ha}$ ) was determined in early December 2013, and all shoots (only live ones, more than $1.5 \mathrm{~m}$ long) were counted per plant. Biometric measurements were performed on ten plants on every plot; the following were measured: plant height and shoot diameter (measurements were made $0.5 \mathrm{~m}$ above the ground level). The plant yield was determined by cutting down entire plants with a chain saw $5-10 \mathrm{~cm}$ above the ground level. Plants obtained from every plot were weighed with BA $300 \mathrm{~K}$ electronic scales (manufactured by Axis) within an accuracy of $0.1 \mathrm{~kg}$ to determine the fresh biomass yield from a plot. The fresh biomass yield and its moisture content were used to calculate the dry matter biomass yield on each plot. During shoot cutting, biomass samples were taken from each of the plots (approximately $5 \mathrm{~kg}$ ) for laboratory analyses. The samples were packed in plastic bags and transported to the laboratory. The biomass moisture content was determined in fresh willow chips in a laboratory, with the drying and weighing method according to PN 80/G04511. Lower heating values of the particular species in the studied combinations were calculated based on the higher heating values (method according to $\mathrm{PN}-81 / \mathrm{G}-04513$, using IKA C 2000 calorimeter) and moisture content determined in a laboratory. The yield energy value $\left(\mathrm{GJ} \mathrm{ha}^{-1}\right)$ was calculated by multiplying the real lower heating values of fresh biomass of the particular species and treatments $\left(\mathrm{GJ} \mathrm{t}^{-1}\right)$ by its yield $\left(\mathrm{t} \mathrm{ha}^{-1}\right)$.

\section{Statistical Analysis}

The experimental data were analyzed statistically using STATISTICA PL software to calculate the mean arithmetic values and standard deviation of the examined traits. 
Homogeneous groups for the examined traits were determined by Tukey's (HSD) multiple-comparison test with the significance level set at $P<0.05$. Principal component analysis (PCA) was applied to evaluate experimental traits. A diagram of the component scores for the first two PCs (F1 and F2) is presented in the form of a biplot.

\section{Weather Conditions}

The weather conditions for all growing seasons are presented in Fig. 1. The year 2010 was generally mild in terms of the average air temperature. Although the first 2 months may have been colder than average, no spring frost was recorded, and the plants had good thermal conditions for growth and development. The amount of rainfall was higher than the multi-year average, both for the whole year and the growing season. However, its distribution was uneven, which undoubtedly hindered plant growth and development. Low rainfall in April 2010 had a particularly negative effect on how well the seedlings of black locust took root. On the other hand, the willow and poplar cuttings fared much better in those conditions. No additional plant watering was applied in the experiment in order to simulate the potential conditions of a commercial plantation. The subsequent growing seasons2011, 2012, and 2013 - were generally warmer compared to the multi-year period, and the total rainfall was rather beneficial for plant growth.

\section{Results}

Survival Rate and the Plant Biometric Features

The number of plants after the fourth growing season varied significantly between species $(P=0.0000)$ and between different combinations of the species and methods of soil enrichment $(P=0.0325)$ (Table 2). The highest survival rate was found for willow (94.8 \%), followed by poplar (92.3\%) and black locust (57.1\%).

The number of shoots on a rootstock ranged from 1.04 to 1.63 , with 1.46 and 1.42 found on average in black locust and willow, respectively, and a smaller number on average (1.1) in poplar (Table 3).

The shoot heights and diameters varied significantly between species $(P=0.0000)$, soil enrichment $(P=0.0000$ and

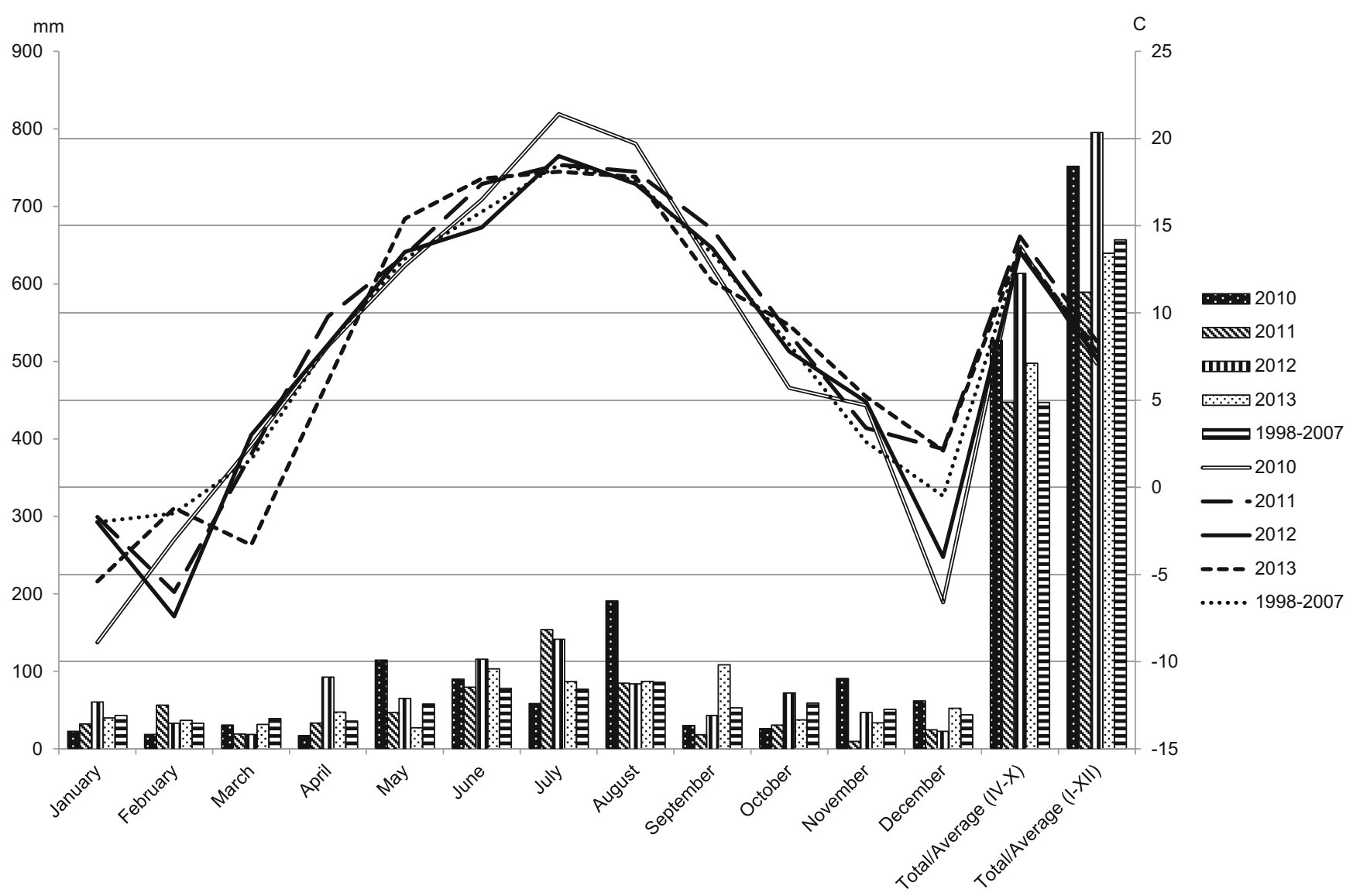

Fig. 1 Weather conditions during the experiment period 2010-2013 and multi-year period 1998-2007. Bars represent precipitation; curves represent air temperatures 
Table 2 Number and survivability of plants after the fourth growing season
Mean \pm standard deviation. Values followed by uppercase letters indicate homogenous groups factor A and factor B. Values followed by lowercase letters indicate homogenous groups interaction AB. Significant at $P<0.05$

\begin{tabular}{|c|c|c|c|}
\hline Species & Soil enrichment procedure & $\begin{array}{l}\text { Number of plants } \\
\left(\text { pieces }^{-1}{ }^{-1}\right)\end{array}$ & Survivability (\%) \\
\hline \multirow[t]{8}{*}{ Black locust } & $\mathrm{C}$ & $5556 \pm 962 d$ & $50.0 \pm 8.7 \mathrm{~d}$ \\
\hline & $\mathrm{L}$ & $6481 \pm 2103 d$ & $58.3 \pm 18.9 \mathrm{~d}$ \\
\hline & $\mathrm{F}$ & $6296 \pm 1156 d$ & $56.7 \pm 10.4 d$ \\
\hline & $\mathrm{LF}$ & $7778 \pm 556 \mathrm{c}$ & $70.0 \pm 5.0 \mathrm{c}$ \\
\hline & M & $6667 \pm 1470 \mathrm{~cd}$ & $60.0 \pm 13.2 \mathrm{~cd}$ \\
\hline & MF & $6111 \pm 692 d$ & $55.0 \pm 8.7 \mathrm{~d}$ \\
\hline & LM & $5370 \pm 642 d$ & $48.3 \pm 5.8 \mathrm{~d}$ \\
\hline & $\mathrm{LMF}$ & $6481 \pm 1398 \mathrm{~d}$ & $58.3 \pm 12.6 \mathrm{~d}$ \\
\hline Mean & & $6343 \pm 1257 \mathrm{~B}$ & $57.1 \pm 11.3 \mathrm{~B}$ \\
\hline \multirow[t]{8}{*}{ Poplar } & $\mathrm{C}$ & $10,556 \pm 0 \mathrm{ab}$ & $95.0 \pm 0.0 \mathrm{ab}$ \\
\hline & $\mathrm{L}$ & $10,185 \pm 1156 a b$ & $91.7 \pm 10.4 \mathrm{ab}$ \\
\hline & $\mathrm{F}$ & $10,185 \pm 321 \mathrm{ab}$ & $91.7 \pm 2.9 \mathrm{ab}$ \\
\hline & $\mathrm{LF}$ & $10,556 \pm 556 a b$ & $95.0 \pm 5.0 \mathrm{ab}$ \\
\hline & M & $10,741 \pm 321 \mathrm{ab}$ & $96.7 \pm 2.9 \mathrm{ab}$ \\
\hline & MF & $10,185 \pm 1156 a b$ & $91.7 \pm 10.4 \mathrm{ab}$ \\
\hline & LM & $9630 \pm 849 b$ & $86.7 \pm 7.6 \mathrm{~b}$ \\
\hline & LMF & $10,000 \pm 556 \mathrm{ab}$ & $90.0 \pm 5.0 \mathrm{ab}$ \\
\hline Mean & & $10,255 \pm 695 \mathrm{~A}$ & $92.3 \pm 6.3 \mathrm{~A}$ \\
\hline \multirow[t]{8}{*}{ Willow } & $\mathrm{C}$ & $9815 \pm 1786 a b$ & $88.3 \pm 16.1 \mathrm{ab}$ \\
\hline & $\mathrm{L}$ & $10,185 \pm 849 a b$ & $91.7 \pm 7.6 \mathrm{ab}$ \\
\hline & $\mathrm{F}$ & $10,926 \pm 321 \mathrm{a}$ & $98.3 \pm 2.9 \mathrm{a}$ \\
\hline & LF & $10,000 \pm 962 \mathrm{ab}$ & $90.0 \pm 8.7 \mathrm{ab}$ \\
\hline & M & $10,556 \pm 556 \mathrm{ab}$ & $95.0 \pm 5.0 \mathrm{ab}$ \\
\hline & MF & $11,111 \pm 0 \mathrm{a}$ & $100.0 \pm 0.0 \mathrm{a}$ \\
\hline & LM & $10,926 \pm 321 \mathrm{a}$ & $98.3 \pm 2.9 \mathrm{a}$ \\
\hline & LMF & $10,741 \pm 321 \mathrm{ab}$ & $96.7 \pm 2.9 \mathrm{ab}$ \\
\hline Mean & & $10,532 \pm 827 \mathrm{~A}$ & $94.8 \pm 7.4 \mathrm{~A}$ \\
\hline \multirow[t]{9}{*}{ Mean for soil enrichment procedure } & $\mathrm{C}$ & $8642 \pm 2548$ & $77.8 \pm 22.9$ \\
\hline & $\mathrm{L}$ & $8951 \pm 2247$ & $80.6 \pm 20.2$ \\
\hline & $\mathrm{F}$ & $9136 \pm 2241$ & $82.2 \pm 20.2$ \\
\hline & $\mathrm{LF}$ & $9444 \pm 1416$ & $85.0 \pm 12.7$ \\
\hline & M & $9321 \pm 2148$ & $83.9 \pm 19.3$ \\
\hline & $\mathrm{MF}$ & $9136 \pm 2423$ & $82.2 \pm 21.8$ \\
\hline & LM & $8642 \pm 2578$ & $77.8 \pm 23.2$ \\
\hline & LMF & $9074 \pm 2115$ & $81.7 \pm 19.0$ \\
\hline & & $P$-value & \\
\hline Species (A) & & 0.0000 & 0.0000 \\
\hline Soil enrichment procedure (B) & & 0.5766 & 0.5766 \\
\hline $\mathrm{AB}$ & & 0.0325 & 0.0325 \\
\hline
\end{tabular}

$P=0.0006$, respectively), and the interactions between them ( $P=0.0397$ and $P=0.0308$, respectively) (Table 3 ). The willows were the tallest (6.94 $\mathrm{m}$ on average). The average plant height was the lowest on the control plot $(6.09 \mathrm{~m})$ and the highest on the LMF plot $(7.94 \mathrm{~m})$. The poplar trees were lower by $0.1 \mathrm{~m}$ on average than the willow plants, and they were included in the same homogeneous group. The poplar height ranged from 6.47 to $7.14 \mathrm{~m}$ in different combinations of soil enrichment. It should be emphasized that the height of the poplar trees was less varied than that of the willows, which is shown by the standard deviation values. The black locusts were the shortest (3.30 $\mathrm{m}$ on average).

The largest shoot diameter was found in poplar $(52.62 \mathrm{~mm}$ on average), while willows were smaller (by $10.36 \mathrm{~mm}$ on 
Table 3 Biometric features of crops after the fourth growing season
Mean \pm standard deviation. Values followed by uppercase letters indicate homogenous groups factor A and factor B. Values followed by lowercase letters indicate homogenous groups interaction AB. Significant at $P<0.05$

\begin{tabular}{|c|c|c|c|c|}
\hline Species & $\begin{array}{l}\text { Soil enrichment } \\
\text { procedure }\end{array}$ & $\begin{array}{l}\text { Number of shoots } \\
\text { (pieces) }\end{array}$ & $\begin{array}{l}\text { Shoot height } \\
\text { (m) }\end{array}$ & $\begin{array}{l}\text { Shoot diameter } \\
(\mathrm{mm})\end{array}$ \\
\hline \multirow[t]{8}{*}{ Black locust } & $\mathrm{C}$ & $1.48 \pm 0.04 \mathrm{ab}$ & $2.85 \pm 0.57 \mathrm{~d}$ & $26.24 \pm 8.23 \mathrm{~d}$ \\
\hline & $\mathrm{L}$ & $1.43 \pm 0.21 \mathrm{ab}$ & $3.46 \pm 0.57 \mathrm{~d}$ & $31.65 \pm 9.25 \mathrm{~cd}$ \\
\hline & $\mathrm{F}$ & $1.37 \pm 0.32 b$ & $2.78 \pm 0.30 \mathrm{~d}$ & $26.93 \pm 5.34 d$ \\
\hline & $\mathrm{LF}$ & $1.47 \pm 0.15 \mathrm{ab}$ & $3.76 \pm 0.32 \mathrm{~d}$ & $37.63 \pm 1.77 b c$ \\
\hline & M & $1.51 \pm 0.12 \mathrm{a}$ & $3.28 \pm 0.37 \mathrm{~d}$ & $31.55 \pm 3.04 \mathrm{~cd}$ \\
\hline & MF & $1.28 \pm 0.07 \mathrm{~b}$ & $3.05 \pm 0.13 \mathrm{~d}$ & $32.78 \pm 3.17 \mathrm{~cd}$ \\
\hline & LM & $1.55 \pm 0.31 \mathrm{a}$ & $3.56 \pm 0.66 \mathrm{~d}$ & $36.08 \pm 5.54 \mathrm{c}$ \\
\hline & LMF & $1.57 \pm 0.15 \mathrm{a}$ & $3.64 \pm 0.27 \mathrm{~d}$ & $34.49 \pm 4.04 \mathrm{c}$ \\
\hline Mean & & $1.46 \pm 0.19 \mathrm{~A}$ & $3.30 \pm 0.51 \mathrm{~B}$ & $32.17 \pm 6.06 \mathrm{C}$ \\
\hline \multirow[t]{8}{*}{ Poplar } & $\mathrm{C}$ & $1.04 \pm 0.07 \mathrm{c}$ & $6.47 \pm 0.22 b c$ & $47.25 \pm 4.69 b$ \\
\hline & $\mathrm{L}$ & $1.10 \pm 0.10 \mathrm{c}$ & $7.14 \pm 0.43 b$ & $50.16 \pm 9.37 b$ \\
\hline & $\mathrm{F}$ & $1.14 \pm 0.15 b c$ & $6.86 \pm 0.41 \mathrm{~b}$ & $54.23 \pm 1.87 \mathrm{ab}$ \\
\hline & $\mathrm{LF}$ & $1.13 \pm 0.12 b c$ & $7.13 \pm 0.12 b$ & $57.90 \pm 3.48 \mathrm{a}$ \\
\hline & M & $1.07 \pm 0.06 \mathrm{c}$ & $6.58 \pm 0.50 \mathrm{bc}$ & $48.01 \pm 4.99 b$ \\
\hline & MF & $1.10 \pm 0.10 \mathrm{c}$ & $6.70 \pm 0.33 b$ & $57.03 \pm 5.25 \mathrm{a}$ \\
\hline & LM & $1.10 \pm 0.00 \mathrm{c}$ & $6.79 \pm 0.38 b$ & $50.10 \pm 3.64 b$ \\
\hline & LMF & $1.10 \pm 0.10 \mathrm{c}$ & $7.04 \pm 0.26 \mathrm{~b}$ & $56.29 \pm 2.25 \mathrm{a}$ \\
\hline Mean & & $1.10 \pm 0.09 \mathrm{~B}$ & $6.84 \pm 0.38 \mathrm{~A}$ & $52.62 \pm 5.79 \mathrm{~A}$ \\
\hline \multirow[t]{8}{*}{ Willow } & $\mathrm{C}$ & $1.37 \pm 0.12 b$ & $6.09 \pm 0.20 \mathrm{c}$ & $36.59 \pm 3.50 \mathrm{c}$ \\
\hline & $\mathrm{L}$ & $1.37 \pm 0.12 b$ & $7.55 \pm 0.42 \mathrm{ab}$ & $49.30 \pm 8.20 b$ \\
\hline & $\mathrm{F}$ & $1.44 \pm 0.22 \mathrm{ab}$ & $6.17 \pm 0.37 b c$ & $38.41 \pm 4.75 \mathrm{c}$ \\
\hline & $\mathrm{LF}$ & $1.34 \pm 0.07 \mathrm{~b}$ & $7.29 \pm 0.55 \mathrm{ab}$ & $42.55 \pm 4.85 b c$ \\
\hline & M & $1.33 \pm 0.32 b$ & $6.31 \pm 0.66 b c$ & $35.63 \pm 2.05 \mathrm{c}$ \\
\hline & MF & $1.37 \pm 0.06 \mathrm{~b}$ & $6.57 \pm 0.67 \mathrm{~b}$ & $40.07 \pm 5.85 \mathrm{c}$ \\
\hline & LM & $1.50 \pm 0.00 \mathrm{a}$ & $7.57 \pm 0.64 \mathrm{ab}$ & $46.57 \pm 12.95 b$ \\
\hline & LMF & $1.63 \pm 0.12 \mathrm{a}$ & $7.94 \pm 0.78 \mathrm{a}$ & $48.97 \pm 9.08 b$ \\
\hline Mean & & $1.42 \pm 0.17 \mathrm{~A}$ & $6.94 \pm 0.84 \mathrm{~A}$ & $42.26 \pm 7.95 \mathrm{~B}$ \\
\hline \multirow{8}{*}{$\begin{array}{l}\text { Mean for soil enrichment } \\
\text { procedure }\end{array}$} & $\mathrm{C}$ & $1.29 \pm 0.21$ & $5.13 \pm 1.75 \mathrm{~B}$ & $36.69 \pm 10.40 \mathrm{~B}$ \\
\hline & $\mathrm{L}$ & $1.30 \pm 0.20$ & $6.05 \pm 2.00 \mathrm{~A}$ & $43.70 \pm 11.92 \mathrm{AB}$ \\
\hline & $\mathrm{F}$ & $1.32 \pm 0.25$ & $5.27 \pm 1.92 \mathrm{~B}$ & $39.86 \pm 12.44 \mathrm{AB}$ \\
\hline & $\mathrm{LF}$ & $1.31 \pm 0.18$ & $6.06 \pm 1.76 \mathrm{~A}$ & $46.03 \pm 9.67 \mathrm{~A}$ \\
\hline & M & $1.30 \pm 0.26$ & $5.39 \pm 1.65 \mathrm{AB}$ & $38.40 \pm 8.04 \mathrm{AB}$ \\
\hline & MF & $1.25 \pm 0.13$ & $5.44 \pm 1.83 \mathrm{AB}$ & $43.29 \pm 11.58 \mathrm{AB}$ \\
\hline & LM & $1.38 \pm 0.26$ & $5.97 \pm 1.91 \mathrm{~A}$ & $44.25 \pm 9.63 \mathrm{AB}$ \\
\hline & LMF & $1.43 \pm 0.27$ & $6.21 \pm 2.01 \mathrm{~A}$ & $46.58 \pm 10.87 \mathrm{~A}$ \\
\hline \multicolumn{5}{|l|}{$P$-value } \\
\hline Species (A) & & 0.0000 & 0.0000 & 0.0000 \\
\hline $\begin{array}{l}\text { Soil enrichment procedure } \\
\text { (B) }\end{array}$ & & 0.3143 & 0.0000 & 0.0006 \\
\hline $\mathrm{AB}$ & & 0.0415 & 0.0397 & 0.0308 \\
\hline
\end{tabular}

average), but the smallest diameters were found in black locust (32.17 $\mathrm{mm}$ on average) (Table 3). Poplar trees developed the thickest shoots on the LF plot $(57.90 \mathrm{~mm})$ and willow on the L plot $(49.30 \mathrm{~mm})$, while black locust shoot diameters ranged from 26.24 to $37.63 \mathrm{~mm}$ on the $\mathrm{C}$ and $\mathrm{LF}$ plots, respectively.
Biomass Yield and Energy Value

The oven dry biomass yield differed significantly between the species $(P=0.0000)$, soil enrichment $(P=0.0000)$, and between their interactions $(P=0.0002)$ (Table 4$)$. The highest average yield was obtained from willow (8.34 odt 
Table 4 Crop yield after the fourth growing season
Mean \pm standard deviation. Values followed by uppercase letters indicate homogenous groups factor A and factor B. Values followed by lowercase letters indicate homogenous groups interaction AB. Significant at $P<0.05$

\begin{tabular}{|c|c|c|c|}
\hline Species & $\begin{array}{l}\text { Soil enrichment } \\
\text { procedure }\end{array}$ & Yield (odt ha ${ }^{-1}$ ) & Yield (odt ha ${ }^{-1}$ year $^{-1}$ ) \\
\hline \multirow[t]{8}{*}{ Black Locust } & $\mathrm{C}$ & $6.54 \pm 1.98 \mathrm{f}$ & $1.63 \pm 0.49 \mathrm{f}$ \\
\hline & $\mathrm{L}$ & $10.89 \pm 2.59 \mathrm{ef}$ & $2.72 \pm 0.65 \mathrm{ef}$ \\
\hline & $\mathrm{F}$ & $8.16 \pm 3.10 \mathrm{f}$ & $2.04 \pm 0.77 \mathrm{f}$ \\
\hline & LF & $21.59 \pm 1.51 \mathrm{~d}$ & $5.40 \pm 0.38 \mathrm{~d}$ \\
\hline & M & $10.78 \pm 1.70 \mathrm{ef}$ & $2.70 \pm 0.43 \mathrm{ef}$ \\
\hline & MF & $9.43 \pm 3.64 \mathrm{ef}$ & $2.36 \pm 0.91 \mathrm{ef}$ \\
\hline & LM & $9.88 \pm 1.21 \mathrm{ef}$ & $2.47 \pm 0.30 \mathrm{ef}$ \\
\hline & LMF & $14.59 \pm 0.83 \mathrm{e}$ & $3.65 \pm 0.21 \mathrm{e}$ \\
\hline Mean & & $11.48 \pm 4.87 \mathrm{~B}$ & $2.87 \pm 1.22 \mathrm{~B}$ \\
\hline \multirow[t]{8}{*}{ Poplar } & $\mathrm{C}$ & $21.91 \pm 2.24 \mathrm{~d}$ & $5.48 \pm 0.56 \mathrm{~d}$ \\
\hline & $\mathrm{L}$ & $36.64 \pm 1.90 \mathrm{~b}$ & $9.16 \pm 0.48 b$ \\
\hline & $\mathrm{F}$ & $36.82 \pm 1.55 b$ & $9.21 \pm 0.39 b$ \\
\hline & $\mathrm{LF}$ & $41.96 \pm 1.84 \mathrm{a}$ & $10.49 \pm 0.46 \mathrm{a}$ \\
\hline & M & $25.51 \pm 3.00 \mathrm{~cd}$ & $6.38 \pm 0.75 \mathrm{~cd}$ \\
\hline & $\mathrm{MF}$ & $34.03 \pm 0.99 b$ & $8.51 \pm 0.25 b$ \\
\hline & LM & $28.60 \pm 2.85 \mathrm{c}$ & $7.15 \pm 0.71 \mathrm{c}$ \\
\hline & LMF & $37.40 \pm 2.29 \mathrm{ab}$ & $9.35 \pm 0.57 \mathrm{ab}$ \\
\hline Mean & & $32.86 \pm 6.78 \mathrm{~A}$ & $8.21 \pm 1.69 \mathrm{~A}$ \\
\hline \multirow[t]{8}{*}{ Willow } & $\mathrm{C}$ & $20.39 \pm 1.16 \mathrm{~d}$ & $5.10 \pm 0.29 \mathrm{~d}$ \\
\hline & $\mathrm{L}$ & $37.28 \pm 4.01 \mathrm{ab}$ & $9.32 \pm 1.00 \mathrm{ab}$ \\
\hline & $\mathrm{F}$ & $36.32 \pm 4.68 b$ & $9.08 \pm 1.17 \mathrm{~b}$ \\
\hline & $\mathrm{LF}$ & $39.32 \pm 1.91 \mathrm{a}$ & $9.83 \pm 0.48 \mathrm{a}$ \\
\hline & $\mathrm{M}$ & $22.41 \pm 6.28 \mathrm{~d}$ & $5.60 \pm 1.57 \mathrm{~d}$ \\
\hline & $\mathrm{MF}$ & $34.18 \pm 1.09 \mathrm{~b}$ & $8.55 \pm 0.27 \mathrm{~b}$ \\
\hline & LM & $35.80 \pm 7.94 b$ & $8.95 \pm 1.98 \mathrm{~b}$ \\
\hline & LMF & $41.20 \pm 3.97 \mathrm{a}$ & $10.30 \pm 0.99 \mathrm{a}$ \\
\hline Mean & & $33.36 \pm 8.27 \mathrm{~A}$ & $8.34 \pm 2.07 \mathrm{~A}$ \\
\hline \multirow[t]{9}{*}{ Mean for soil enrichment procedure } & $\mathrm{C}$ & $16.28 \pm 7.51 \mathrm{D}$ & $4.07 \pm 1.88 \mathrm{D}$ \\
\hline & $\mathrm{L}$ & $28.27 \pm 13.29 \mathrm{~B}$ & $7.07 \pm 3.32 \mathrm{~B}$ \\
\hline & $\mathrm{F}$ & $27.10 \pm 14.50 \mathrm{~B}$ & $6.78 \pm 3.63 \mathrm{~B}$ \\
\hline & LF & $34.29 \pm 9.71 \mathrm{~A}$ & $8.57 \pm 2.43 \mathrm{~A}$ \\
\hline & $\mathrm{M}$ & $19.57 \pm 7.62 \mathrm{C}$ & $4.89 \pm 1.91 \mathrm{C}$ \\
\hline & $\mathrm{MF}$ & $25.88 \pm 12.49 \mathrm{BC}$ & $6.47 \pm 3.12 \mathrm{BC}$ \\
\hline & LM & $24.76 \pm 12.35 \mathrm{BC}$ & $6.19 \pm 3.09 \mathrm{BC}$ \\
\hline & LMF & $31.06 \pm 12.68 \mathrm{AB}$ & $7.77 \pm 3.17 \mathrm{AB}$ \\
\hline & & $P$-value & \\
\hline Species (A) & & 0.0000 & 0.0000 \\
\hline Soil enrichment procedure (B) & & 0.0000 & 0.0000 \\
\hline $\mathrm{AB}$ & & 0.0002 & 0.0002 \\
\hline
\end{tabular}

$\mathrm{ha}^{-1}$ year $^{-1}$ ); poplar yield was similar (8.21 odt ha ${ }^{-1}$ year $^{-1}$ ), whereas the yield of black locust was 2.87 odt ha $^{-1}$ year ${ }^{-1}$. The highest yield of poplar was obtained on the LF plot (10.49 odt ha ${ }^{-1}$ year $^{-1}$ ), although it was 39.2 and $47.8 \%$ lower on the $\mathrm{M}$ and $\mathrm{C}$ plots, respectively. The highest yield of willow was obtained on the LMF plot (10.3 odt ha ${ }^{-1}$ year $\left.^{-1}\right)$. This was included in the same homogeneous group as the highest yield of poplar and willow from the LF plot. The lowest yield of willow was obtained on the $\mathrm{C}$ and $\mathrm{M}$ plots (by 50.5 and $45.6 \%$ ), respectively, than the highest yield of the species. The highest yield of black locust was obtained on the LF plot (5.4 odt ha ${ }^{-1}$ year $\left.^{-1}\right)$. 
The lower heating value of biomass was significantly differentiated by the species $(P=0.0000)$, while soil enrichment and interactions between factors were insignificant ( $P=0.2129$ and $P=0.6321$, respectively) (Table 5). However, the energy value of the biomass yield was significantly differentiated by the species $(P=0.0000)$, soil enrichment
( $P=0.0000)$, and between their interactions $(P=0.0002)$. The highest yield energy value was found for poplar (176.7 GJ $\mathrm{ha}^{-1}$ year $^{-1}$ ) grown on the LF plot. It was $11-48 \%$ lower on other poplar plots. The willow yield energy value was $1-51 \%$ lower than the highest value achieved in the experiment, and it ranged from 175.1 to $87.4 \mathrm{GJ} \mathrm{ha}^{-1}$ year $^{-1}$. The energy value

Table 5 Lower heating value and biomass energy yield value

\begin{tabular}{|c|c|c|c|c|}
\hline Species & Soil enrichment procedure & $\begin{array}{l}\text { Lower heating value } \\
\left(\mathrm{GJ} \mathrm{t}^{-1}\right)\end{array}$ & $\begin{array}{l}\text { Yield energy value } \\
\left(\mathrm{GJ} \mathrm{ha}^{-1}\right)\end{array}$ & $\left(\mathrm{GJ} \mathrm{ha}^{-1}\right.$ year $\left.^{-1}\right)$ \\
\hline \multirow[t]{8}{*}{ Black locust } & $\mathrm{C}$ & $10.10 \pm 0.15$ & $114.4 \pm 34.7 f$ & $28.6 \pm 8.7 f$ \\
\hline & $\mathrm{L}$ & $10.12 \pm 0.01$ & $190.0 \pm 45.2 \mathrm{ef}$ & $47.5 \pm 11.3 \mathrm{ef}$ \\
\hline & $\mathrm{F}$ & $10.27 \pm 0.14$ & $144.1 \pm 54.4 \mathrm{f}$ & $36.0 \pm 13.6 f$ \\
\hline & LF & $10.19 \pm 0.14$ & $379.3 \pm 25.7 d$ & $94.8 \pm 6.4 \mathrm{~d}$ \\
\hline & M & $10.16 \pm 0.01$ & $189.6 \pm 29.7 \mathrm{ef}$ & $47.4 \pm 7.4 \mathrm{ef}$ \\
\hline & $\mathrm{MF}$ & $10.12 \pm 0.08$ & $165.9 \pm 64.3 f$ & $41.5 \pm 16.1 \mathrm{f}$ \\
\hline & $\mathrm{LM}$ & $10.21 \pm 0.15$ & $173.4 \pm 21.3 \mathrm{ef}$ & $43.3 \pm 5.3 \mathrm{ef}$ \\
\hline & LMF & $10.12 \pm 0.08$ & $256.1 \pm 15.4 \mathrm{e}$ & $64.0 \pm 3.8 \mathrm{e}$ \\
\hline Mean & & $10.16 \pm 0.11 \mathrm{~A}$ & $201.6 \pm 85.6 \mathrm{~B}$ & $50.4 \pm 21.4 \mathrm{~B}$ \\
\hline \multirow[t]{8}{*}{ Poplar } & $\mathrm{C}$ & $7.48 \pm 0.05$ & $370.1 \pm 37.6 \mathrm{~d}$ & $92.5 \pm 9.4 \mathrm{~d}$ \\
\hline & $\mathrm{L}$ & $7.46 \pm 0.15$ & $617.9 \pm 30.8 b$ & $154.5 \pm 7.7 \mathrm{~b}$ \\
\hline & $\mathrm{F}$ & $7.45 \pm 0.09$ & $620.4 \pm 26.2 b$ & $155.1 \pm 6.5 b$ \\
\hline & LF & $7.45 \pm 0.07$ & $706.7 \pm 32.1 \mathrm{a}$ & $176.7 \pm 8.0 \mathrm{a}$ \\
\hline & M & $7.46 \pm 0.07$ & $429.5 \pm 49.2 \mathrm{~cd}$ & $107.4 \pm 12.3 \mathrm{~cd}$ \\
\hline & MF & $7.38 \pm 0.07$ & $571.2 \pm 17.8 \mathrm{~b}$ & $142.8 \pm 4.5 b$ \\
\hline & LM & $7.42 \pm 0.03$ & $481.0 \pm 47.9 \mathrm{c}$ & $120.3 \pm 12.0 \mathrm{c}$ \\
\hline & LMF & $7.43 \pm 0.04$ & $629.1 \pm 38.8 \mathrm{ab}$ & $157.3 \pm 9.7 \mathrm{ab}$ \\
\hline Mean & & $7.44 \pm 0.07 \mathrm{C}$ & $553.2 \pm 113.9 \mathrm{~A}$ & $138.3 \pm 28.5 \mathrm{~A}$ \\
\hline \multirow[t]{8}{*}{ Willow } & $\mathrm{C}$ & $8.51 \pm 0.04$ & $349.8 \pm 19.1 d$ & $87.4 \pm 4.8 \mathrm{~d}$ \\
\hline & $\mathrm{L}$ & $8.44 \pm 0.07$ & $635.5 \pm 68.5 \mathrm{ab}$ & $158.9 \pm 17.1 \mathrm{ab}$ \\
\hline & $\mathrm{F}$ & $8.51 \pm 0.04$ & $620.5 \pm 81.1 \mathrm{~b}$ & $155.1 \pm 20.3 b$ \\
\hline & $\mathrm{LF}$ & $8.40 \pm 0.06$ & $669.8 \pm 31.5 \mathrm{a}$ & $167.4 \pm 7.9 \mathrm{a}$ \\
\hline & M & $8.46 \pm 0.12$ & $381.7 \pm 106.1 \mathrm{~d}$ & $95.4 \pm 26.5 d$ \\
\hline & $\mathrm{MF}$ & $8.41 \pm 0.05$ & $581.4 \pm 18.9 \mathrm{~b}$ & $145.3 \pm 4.7 \mathrm{~b}$ \\
\hline & $\mathrm{LM}$ & $8.35 \pm 0.05$ & $607.6 \pm 133.6 b$ & $151.9 \pm 33.4 \mathrm{~b}$ \\
\hline & LMF & $8.37 \pm 0.02$ & $700.5 \pm 68.2 \mathrm{a}$ & $175.1 \pm 17.0 \mathrm{a}$ \\
\hline Mean & & $8.43 \pm 0.08 \mathrm{~B}$ & $568.3 \pm 140.1 \mathrm{~A}$ & $142.1 \pm 35.0 \mathrm{~A}$ \\
\hline \multirow[t]{8}{*}{ Mean for soil enrichment procedure } & $\mathrm{C}$ & $8.70 \pm 1.15$ & $278.1 \pm 126.1 \mathrm{C}$ & $69.5 \pm 31.5 \mathrm{C}$ \\
\hline & $\mathrm{L}$ & $8.67 \pm 1.17$ & $481.2 \pm 222.8 \mathrm{~B}$ & $120.3 \pm 55.7 \mathrm{~B}$ \\
\hline & $\mathrm{F}$ & $8.74 \pm 1.23$ & $461.7 \pm 243.5 B$ & $115.4 \pm 60.9 \mathrm{~B}$ \\
\hline & LF & $8.68 \pm 1.21$ & $585.3 \pm 157.5 \mathrm{~A}$ & $146.3 \pm 39.4 \mathrm{~A}$ \\
\hline & M & $8.69 \pm 1.18$ & $333.6 \pm 125.4 \mathrm{C}$ & $83.4 \pm 31.4 \mathrm{C}$ \\
\hline & $\mathrm{MF}$ & $8.64 \pm 1.2$ & $439.5 \pm 208.1 \mathrm{BC}$ & $109.9 \pm 52.0 \mathrm{BC}$ \\
\hline & LM & $8.66 \pm 1.23$ & $420.7 \pm 206.3 \mathrm{BC}$ & $105.2 \pm 51.6 \mathrm{BC}$ \\
\hline & LMF & $8.64 \pm 1.18$ & $528.5 \pm 210.5 \mathrm{AB}$ & $132.1 \pm 52.6 \mathrm{AB}$ \\
\hline \multicolumn{5}{|l|}{$P$-value } \\
\hline Species (A) & & 0.0000 & 0.0000 & 0.0000 \\
\hline Soil enrichment procedure (B) & & 0.2129 & 0.0000 & 0.0000 \\
\hline $\mathrm{AB}$ & & 0.6321 & 0.0002 & 0.0002 \\
\hline
\end{tabular}

Mean \pm standard deviation. Values followed by uppercase letters indicate homogenous groups factor A and factor B. Values followed by lowercase letters indicate homogenous groups interaction $\mathrm{AB}$. Significant at $P<0.05$ 
for black locust was the lowest, and it ranged from 94.8 to 28.6 GJ ha ${ }^{-1}$ year $^{-1}$.

Principal component analysis revealed that the variability of the plant species under study can be $80.5 \%$ explained by the first principal component (F1) through strong correlation of the yield structure features (number of plants, height, diameter), biomass yield, lower heating value, and the yield energy value. The number of shoots was the second component (F2); it contributed another $13.5 \%$ to the explanation of the variability of the plots under study (Table 6).

The biplot graph clearly shows the separation of the species under study (three separate "point isles") (Fig. 2). In the top lefthand corner, there are points assigned to poplar on various enrichment plots, which indicates that the species was characterized by a small number of shoots and by the lowest lower heating value, high yield-forming parameters, and a high yield energy value. The points assigned to willow are in the bottom left-hand corner, which means that the species had a high yielding and energy potential, and it differed from poplar by having a large number of shoots. Black locust forms the third separate group of points. As well as having the highest lower heating value, the yield and yield energy values were the lowest and were considerably below the potential of willow and poplar.

\section{Discussion}

Since growing a specific SRWC crop offers a high yield and potentially high profits, the yields of different plant species are of key importance. Apart from productivity in a specific year or harvest cycle, the yield is affected by the presence of pathogens and the plant vigor associated with it. No significant infestation of the plants under study by diseases or pests was recorded during the four growing seasons. However, observations must be conducted because the literature data mentioned considerable damage caused by pests and diseases on SRWC plantations [2, 28-30]. It should be noted that

Table 6 Row factorial loadings

\begin{tabular}{lrr}
\hline Traits & F1 & \multicolumn{1}{l}{ F2 } \\
\hline Number of plants (NoP) & -0.93 & -0.09 \\
Plant height (PH) & -0.97 & -0.12 \\
Stem diameter (D) & -0.91 & 0.22 \\
Number of shoots (NoS) & 0.54 & -0.83 \\
Yield of dry biomass (YB) & -0.96 & -0.23 \\
Yield energy value (YE) & -0.96 & -0.24 \\
Lower heating value (LHV) & 0.95 & -0.25 \\
Eigenvalue $\lambda_{i}$ & 5.64 & 0.95 \\
Share (\%) & 80.54 & 13.52 \\
\hline
\end{tabular}

Italics indicate significant coefficients. Significant at $P<0.05$
SRWC plantations are sometimes eaten by wild animals (deer, wisent, and elk) [31]. In our experiment, losses in black locust caused by wild animals and low precipitation in the setup year of the experiment resulted in a decrease in the total yielding potential.

The methods of soil enrichment used in the experiment resulted in yield diversification (from 1.6 to $10.5 \mathrm{odt}$ $\mathrm{ha}^{-1}$ year ${ }^{-1}$ ) among different species and soil enrichment procedures. Such a wide yield diversity for different species indicates that there is a need for further studies to confirm and verify the data in subsequent harvest rotations. On the other hand, it must be emphasized that other publications have confirmed the significant diversification of yield depending on the SRWC species and the amount of fertilizers applied. On a willow plantation in sandy soil in Denmark, the average annual biomass production ranged from $8.7 \mathrm{odt} \mathrm{ha}^{-1}$ year $^{-1}$ in the control up to $11.9 \mathrm{odt} \mathrm{ha}^{-1}$ year $^{-1}$ fertilized with $60 \mathrm{~kg} \mathrm{~N} \mathrm{ha}^{-1}$ year $^{-1}$ [22]. Similarly, a high willow yield was achieved in the USA from 8.4 on control plots to between 9 and 11.6 odt ha $^{-1}$ year $^{-1}$ with different applications of NPK [32]. In a study conducted in central Sweden [1], the yield for two varieties of willow obtained on the plots under control conditions was similar to the willow yield obtained on the control plot in this study. The willow yield increased significantly depending on the intensity of fertilization and its strategy. In the economic treatment, the average yield was $9.3 \mathrm{odt} \mathrm{ha}^{-1}$ year $^{-1}$, whereas it was $10.8 \mathrm{odt}$ $\mathrm{ha}^{-1}$ year ${ }^{-1}$ in the normal treatment and 13.2 odt ha $^{-1}$ year ${ }^{-1}$ for the intensive treatment [1]. Also, high yield for four willow clones (average 14.1 odt ha $^{-1}$ year $^{-1}$ ) grown on very good soil and fertilized was obtained in a 4-year harvest cycle [33].

A high yield of poplar biomass was obtained in a 4-year harvest cycle in Canada for the clone NM P. maximowiczii $\times P$. nigra (NM6) (18.0 odt ha year $^{-1}$ ) [8]. A similar yield was found for poplar grown in the same cutting cycle in Italy [34]. A very high yield of six genotypes of poplar in three consecutive 2-year harvest rotations was obtained by Sabatti et al. [35]. Biomass production differed significantly among the rotations, starting from $16 \mathrm{odt} \mathrm{ha}^{-1}$ year $^{-1}$ in the first year, peaking at $20 \mathrm{odt} \mathrm{ha}^{-1}$ year $^{-1}$ in the second, and decreasing to 17 odt ha $^{-1}$ year $^{-1}$ in the third rotation. However, other authors have reported that seven poplar clones of Populus $\times$ canadensis and seven of the Populus deltoides grown in Italy did not give such a high yield [36]. The yield of poplar obtained in other studies also varied depending on the climatic conditions, the type of soil, species, and clone, harvest rotation, age of the plantation, level of fertilization, and other agricultural procedures [37-39].

Black locust is an important species in land reclamation and potentially as a species to produce biomass on poorquality soils. In Hungary, black locust obtained in a 5-year harvest rotation at a density of about 22,000 plants per hectare gave a yield of 6.5 odt ha ${ }^{-1}$ year $^{-1}$ [14]. However, when grown at two lower densities, the yields were 33-51\% lower. 


\section{Biplot}

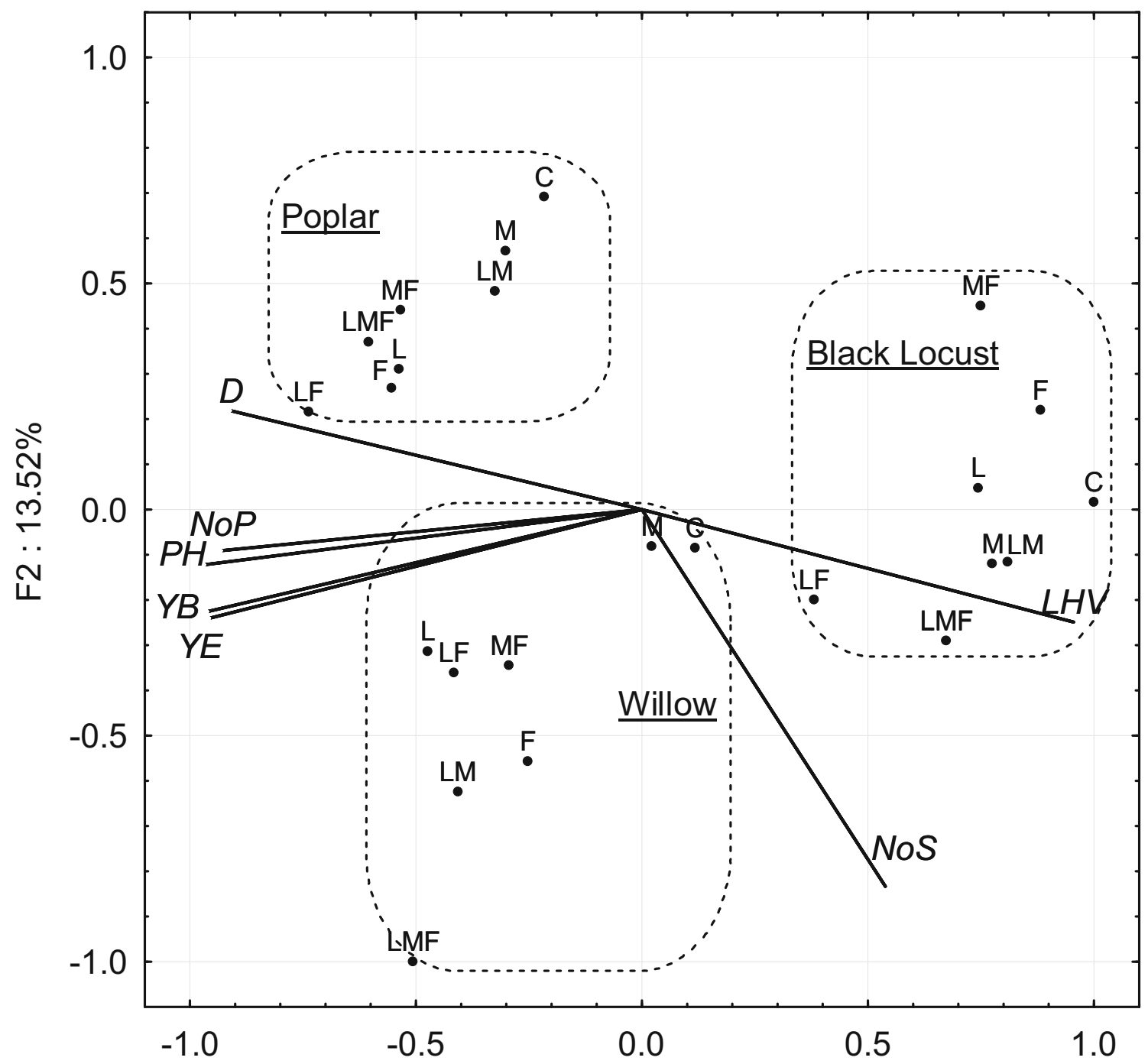

F1: $80.54 \%$

Fig. 2 Biplot for analyzed data. $D$ diameter, $N o P$ number of plants, $P H$ height, $Y B$ biomass yield, $Y E$ energy yield, $N o S$ number of shoots, $L H V$ lower heating value

Gruenewald et al. [40] conducted a study with black locust planted on poor-quality soil at a former brown coal opencast mine. The average yield in a 3-year rotation was approximately 4 and 6 odt ha $^{-1}$ year $^{-1}$ in a 6 -year rotation. Black locust gave a higher yield than poplar and willow despite the low quality of soil and disadvantageous climatic conditions. Moreover, these results show that black locust adapts well to sandy sites which are poor in nutrients, which was confirmed in later studies [41].

The energy value of the yield in this experiment ranged from 28.6 to $176.7 \mathrm{GJ} \mathrm{ha}^{-1}$ year $^{-1}$. A high energy value for the poplar yield of $188 \mathrm{GJ} \mathrm{ha}^{-1}$ year $^{-1}$ was achieved in the production of the crop in a 2-year harvest cycle [42]. On the other hand, the energy value of the poplar yield obtained in extensive cultivation in a 4-year harvest rotation was much lower (70.9 GJ ha ${ }^{-1}$ year $^{-1}$ ) [43]. This was confirmed by Dillen et al. [39] who showed that the energy value of the yield of poplar grown on degraded land was about 91.8 GJ $\mathrm{ha}^{-1}$ year ${ }^{-1}$. The values were comparable with the energy value of the poplar yield obtained on the control plot in this experiment. In a study conducted in Poland on an experimental willow plantation, the yield energy value was high and lay within a wide range (from 188 to $349 \mathrm{GJ} \mathrm{ha}^{-1}$ year $^{-1}$ ) [33, 44]. Furthermore, the energy value of the yield of willow grown on a commercial plantation in a 3-year cycle ranged from 46.3 to $242.5 \mathrm{GJ} \mathrm{ha}^{-1}$ year $^{-1}$ [4]. The mean net energy from willow plantations in Sweden was approximately $170 \mathrm{GJ} \mathrm{ha}^{-1}$ year $^{-1}$ [45]. This may even exceed $200 \mathrm{GJ} \mathrm{ha}^{-1}$ year $^{-1}$ when waste water is used for willow plantation irrigation [46]. A positive 
effect of using sludge was confirmed in a study conducted in Canada, in which the energy value of the willow yield ranged from 73 to $290 \mathrm{GJ} \mathrm{ha}^{-1}$ year $^{-1}$ with a sludge dose of 0 and $300 \mathrm{~kg} \mathrm{~N} \mathrm{ha}^{-1}$, respectively [47].

\section{Conclusions}

This study found considerable diversity in the productivity and energy value of the SRWC yield not only between species but also depending on the soil enrichment methods and the interactions between these factors. Principal component analysis clearly showed the distinction between the three species under study. It was shown that soil enrichment by using lignin, mycorrhiza, and mineral fertilization can significantly increase the productivity of SRWC species compared to control plots. It must be emphasized that various combinations of mineral fertilization, mycorrhiza, and lignin contributed to a threefold increase in the yield of black locust compared to the control plot and more than a twofold increase for poplar and willow. Importantly, the use of lignin in combination with mineral fertilizers resulted in an increase in the yield by 8 $14 \%$ compared to mineral fertilizers alone for willow and poplar and in a nearly twofold increase for black locust. In conclusion, these findings indicate the possibility of increasing productivity and energy value of the SRWC yield on poor soils, with low usability for edible crops, by choosing the right species of woody crops and the method of soil enrichment.

Acknowledgments This work has been financed by the strategic program of the National (Polish) Centre for Research and Development (NCBiR): "Advanced Technologies for Energy Generation. Task 4: Elaboration of Integrated Technologies for the Production of Fuels and Energy from Biomass, Agricultural Waste and other Waste Materials".

Open Access This article is distributed under the terms of the Creative Commons Attribution License which permits any use, distribution, and reproduction in any medium, provided the original author(s) and the source are credited.

\section{References}

1. Aronsson P, Rosenqvist H, Dimitriou I (2014) Impact of nitrogen fertilization to short-rotation willow coppice plantations grown in Sweden on yield and economy. Bioenergy Res. doi:10.1007/ s12155-014-9435-7

2. Larsen SU, Jørgensen U, Lærke PE (2014) Willow yield is highly dependent on clone and site. Bioenergy Res. doi:10.1007/s12155014-9463-3

3. Stolarski M, Szczukowski S, Tworkowski J, Wróblewska H, Krzyżaniak M (2011) Short rotation willow coppice biomass as an industrial and energy feedstock. Ind Crop Prod 33:217-223

4. Stolarski MJ, Krzyżaniak M, Tworkowski J, Szczukowski S, Gołaszewski J (2014) Energy intensity and energy ratio in producing willow chips as feedstock for an integrated biorefinery. Biosyst Eng 123:19-28
5. Faber A, Pudełko R, Borek R, Borzecka-Walker M, Syp A, Krasuska E, Mathiou P (2012) Economic potential of perennial energy crops in Poland. J Food Agric Environ 10(3-4):1178-1182

6. Volk TA, Abrahamson LP, Nowak CA, Smart LB, Tharakan PJ, White EH (2006) The development of short-rotation willow in the northeastern United States for bioenergy and bioproducts, agroforestry and phytoremediation. Biomass Bioenergy 30(8-9):715-727

7. Serapiglia MJ, Cameron KD, Stipanovic AJ, Abrahamson LP, Volk TA, Smart LB (2013) Yield and woody biomass traits of novel shrub willow hybrids at two contrasting sites. Bioenergy Res 6:533-546

8. Labrecque M, Teodorescu TL (2005) Field performance and biomass production of 12 willow and poplar clones in short-rotation coppice in southern Quebec (Canada). Biomass Bioenergy 29(1):1-9

9. González-Garćia S, Gasol CM, Gabarrell X, Rieradevall J, Teresa Moreira M, Feijoo G (2010) Environmental profile of ethanol from poplar biomass as transport fuel in Southern Europe. Renew Energy 35:1014-1023

10. Spinelli R, Nati C, Magagnotti N (2009) Using modified foragers to harvest short-rotation poplar plantations. Biomass Bioenergy 33: 817-821

11. Aravanopoulos FA (2010) Breeding of fast growing forest tree species for biomass production in Greece. Biomass Bioenergy 34:15311537

12. AEBIOM (2013) European bioenergy outlook 2013. Statistical report, Brussels, $p 121$

13. Rédei K, Osváth-Bujtás Z, Veperdi I (2008) Black locust (Robinia pseudoacacia L.) improvement in Hungary: a review. Acta Silv Lign Hung 4:127-132

14. Rédei K, Veperdi I (2009) The role of black locust (Robinia pseudoacacia L.) in establishment of short-rotation energy plantations in Hungary. Int J Hortic Sci 15(3):41-44

15. Stolarski M, Szczukowski S, Tworkowski J, Klasa A (2008) Productivity of seven clones of willow coppice in annual and quadrennial cutting cycles. Biomass Bioenergy 32(12):1227-1234

16. Tharakan PJ, Volk TA, Nowak CA, Abrahamson LP (2005) Morphological traits of 30 willow clones and their relationship to biomass production. Can J For Res 35(2):421-431

17. Stolarski MJ, Szczukowski S, Tworkowski J, Klasa A (2011) Willow biomass production under conditions of low-input agriculture on marginal soils. For Ecol Manag 262:1558-1566

18. Wilkinson JM, Evans EJ, Bilsborrow PE, Wright C, Hewison WO, Pilbeam DJ (2007) Yield of willow cultivars at different planting densities in a commercial short rotation coppice in the north of England. Biomass Bioenergy 31(7):469-474

19. Lindroth A, Bath A (1999) Assessment of regional willow coppice yield in Sweden on basis of water availability. For Ecol Manag 121(1-2):57-65

20. Tahvanainen L, Rytkonen VM (1999) Biomass production of Salix viminalis in southern Finland and the effect of soil properties and climate conditions on its production and survival. Biomass Bioenergy 16(2): 103-117

21. Weih M (2004) Intensive short rotation forestry in boreal climates: present and future perspectives. Can J For Res Rev Can Rech Forestiere 34(7):1369-1378

22. Sevel L, Nord-Larsen T, Ingerslev M, Jørgensen U, RaulundRasmussen K (2014) Fertilization of SRC willow, I: biomass production response. Bioenergy Res 7:319-328

23. Mortensen J, Nielsen KH, Jorgensen U (1998) Nitrate leaching during establishment of willow (Salix viminalis) on two soil types and at two fertilization levels. Biomass Bioenergy 15(6):457-466

24. Aronsson PG, Bergstrom LF (2001) Nitrate leaching from lysimetergrown short-rotation willow coppice in relation to $\mathrm{N}$-application, irrigation and soil type. Biomass Bioenergy 21(3):155-164

25. Quaye AK, Volk TA, Hafner S, Leopold DJ, Schirmer C (2011) Impacts of paper sludge and manure on soil and biomass production of willow. Biomass Bioenergy 35(7):2796-2806 
26. Rooney DC, Killham K, Bending GD, Baggs E, Weih M, Hodge A (2009) Mycorrhizas and biomass crops: opportunities for future sustainable development. Trends Plant Sci 14:542-549

27. Smolarski N (2012) High-value opportunities for lignin: unlocking its potential. Frost \& Sullivan, http://www.greenmaterials.fr/wpcontent/uploads/2013/01/High-value-Opportunities-for-LigninUnlocking-its-Potential-Market-Insights.pdf. Accessed 21 Nov 2014

28. Royle DJ, Ostry ME (1995) Disease and pest control in the bioenergy crops poplar and willow. Biomass Bioenergy 9(1/5):69-79

29. Peacock L, Harris J, Powers S (2004) Effects of host variety on blue willow beetle Phratora vulgatissima performance. Ann Appl Biol 144(1):45-52

30. Lindegaard KN, Carter MM, McCracken A, Shield I, MacAlpineW H-JM, Valentine J, Larsson S (2011) Comparative trials of elite Swedish and UK biomass willow varieties 2001-2010. Asp Appl Biol 112:57-65

31. Stolarski MJ, Krzyżaniak M, Szczukowski S, Tworkowski J, Bieniek A (2013) Dendromass derived from agricultural land as energy feedstock. Pol J Environ Stud 22(2):511-520

32. Adegbidi HG, Briggs RD, Volk TA, White EH, Abrahamson LP (2003) Effect of organic amendments and slow-release nitrogen fertilizer on willow biomass production and soil chemical characteristics. Biomass Bioenergy 25(4):389-398

33. Stolarski MJ, Szczukowski S, Tworkowski J, Klasa A (2013) Yield, energy parameters and chemical composition of short-rotation willow biomass. Ind Crop Prod 46:60-65

34. Guidi W, Tozzini C, Bonari E (2009) Estimation of chemical traits in poplar short-rotation coppice at stand level. Biomass Bioenergy 33(12):1703-1709

35. Sabatti M, Fabbrini F, Harfouche A, Beritognolo I, Mareschi L, Carlini M, Paris P, Scarascia-Mugnozza G (2014) Evaluation of biomass production potential and heating value of hybrid poplar genotypes in a short-rotation culture in Italy. Ind Crop Prod 61:62-73

36. Bergante S, Facciotto G (2011) Nine years measurements in Italian SRC trial in 14 poplar and 6 willow clones. 19th European Biomass
Conference and Exhibition, Berlin, Germany 6-10 June 2011, conference proceedings

37. Laureysens I, Pellis A, Willems J, Ceulemans R (2005) Growth and production of a short rotation coppice culture of poplar. III. Second rotation results. Biomass Bioenergy 29:10-21

38. Christersson L (2010) Wood production potential in poplar plantations in Sweden. Biomass Bioenergy 34:1289-1299

39. Dillen SY, Djomo SN, Al Afas N, Vanbeveren S, Ceulemans R (2013) Biomass yield and energy balance of a short rotation poplar coppice with multiple clones on degraded land during 16 years. Biomass Bioenergy 56:157-165

40. Gruenewald H, Brandt BKV, Schneider BU, Bens O, Kendzia G, Hüttl RF (2007) Agroforestry systems for the production of woody biomass for energy transformation purposes. Ecol Eng 29:319-328

41. Gruenewald H, Böhm C, Quinkenstein A, Grundmann P, Eberts J, von Wühlisch G (2009) Robinia pseudoacacia L.: a lesser known tree species for biomass production. Bioenergy Res 2:123-133

42. Manzone M, Airoldi G, Balsari P (2009) Energetic and economic evaluation of a poplar cultivation for the biomass production in Italy. Biomass Bioenergy 33:1258-1264

43. Walle IV, Van Camp N, Van De Casteele L, Verheyen K, Lemeur R (2007) Short-rotation forestry of birch, maple, poplar and willow in Flanders (Belgium) II. Energy production and $\mathrm{CO}_{2}$ emission reduction potential. Biomass Bioenergy 31(5):276-283

44. Stolarski M (2009) Agrotechnical and economic aspects of biomass production from willow coppice (Salix spp.) as an energy source (in Polish). University of Warmia and Mazury in Olsztyn, Olsztyn

45. Börjesson PII (1996) Energy analysis of biomass production and transportation. Biomass Bioenergy 11(4):305-318

46. Börjesson P, Berndes G (2006) The prospects for willow plantations for wastewater treatment in Sweden. Biomass Bioenergy 30(5):428-438

47. Labrecque M, Teodorescu TI, Daigle S (1997) Biomass productivity and wood energy of Salix species after 2 years growth in SRIC fertilized with wastewater sludge. Biomass Bioenergy 12(6):409417 